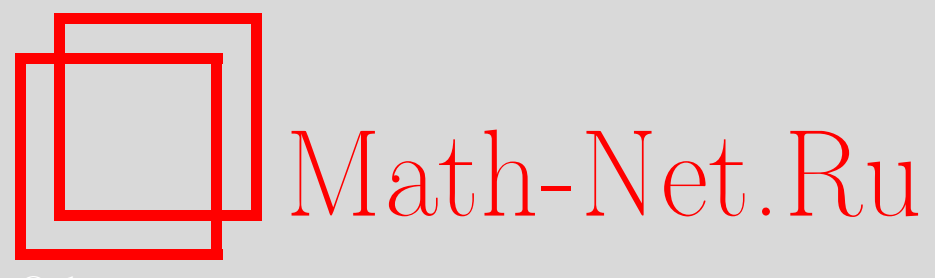

Д. Чибисов, Элементарное доказательство закона больших чисел, Квант, 2019, номер 5, 10-16

DOI: https://doi.org/10.4213/kvant20190502

Использование Общероссийского математического портала Math-Net.Ru подразумевает, что вы прочитали и согласны с пользовательским соглашением http://www . mathnet.ru/rus/agreement

Параметры загрузки:

IP: 54.172 .240 .79

26 апреля 2023 г., 15:46:30

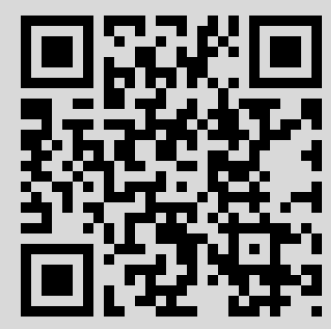




\section{Элементарное доказательство закона больших чисел}

Д.ЧИБИСОВ

\section{1. Введение}

Закон больших чисел (далее ЗБЧ) фундаментальный закон теории вероятностей (далее ТВ), связывающий математическое понятие вероятности с явлениями реальной жизни. Первоначальное развитие ТВ связано с практикой азартных игр. Например, в игре двух игроков с двумя равновероятными исходами ставки игроков, естественно, должны быть равны. (Обычно в ТВ такая игра иллюстрируется бросанием монеты, но практически это может быть чет-нечет в рулетке или игра фараон, описанная в «Пиковой даме», и т.п.) А предположим, я играю на то, что число очков, которое выпадет при бросании игральной кости, делится на 3. Какова будет справедливая ставка? Поскольку я выигрываю в 2 случаях из 6 возможных, то вероятность моего выигрыша равна $\frac{1}{3}$. Нетрудно понять, что справедливая ставка должна быть 2 руб. против моего 1 руб. Действительно, тогда в большой серии игр я выигрываю по 2 руб. в примерно $\frac{1}{3}$ игр, т.е. по $\frac{2}{3}$ в среднем на игру, а мой оппонент - по 1 руб. в $\frac{2}{3}$ игр, т.е. те же $\frac{2}{3}$ на игру.

Обратим внимание на последнюю фразу, в которой подразумевается, как само собой разумеющееся, что относительная частота события должна быть приближенно равна его вероятности. Эта закономерность, которую можно назвать «эмпирическим законом больших чисел», была особенно хорошо известна заядлым игрокам, быстро улавливавшим отклонения от

DOI: https://doi.org/10.4213/kvant20190502 нее и искавшим их причину либо в собственном неправильном подсчете вероятности, либо в шулерстве оппонента.

C развитием ТВ встала задача вывести этот закон из принципов, лежащих в основе ТВ (о которых будет рассказано ниже). Это, с одной стороны, дало бы закону научное обоснование, а с другой - подтвердило бы правильность положений, лежащих в основе самой ТВ, тем, что вытекающие из них следствия соответствуют реальности.

Доказательство ЗБЧ было дано швейцарским математиком Яковом Бернулли в книге «Искусство предположений» («Ars Conjectandi»), опубликованной в 1713 году (см. [1]).

Впоследствии ЗБЧ сыграл важную роль, например, в возникновении генетики, когда из соотношения частот наследования признаков в опытах Менделя, близкого к 3:1, было сделано предположение, что признаки наследуются с вероятностями $\frac{3}{4}$ и $\frac{1}{4}$, и объяснение механизма этого явления составило один из законов Менделя.

В настоящей статье мы приводим элементарное доказательство ЗБЧ, близкое к доказательству Я.Бернулли, во всяком случае, не использующее никаких полученных позднее результатов. В учебнике [2] (как и во всех современных курсах ТВ) ЗБЧ доказывается с помощью неравенства Чебышёва, но оно было открыто в XIX веке, а интерес данной статьи - доказательство средствами, доступными Бернулли.

Мы не предполагаем от читателя никакux познаний в ТВ. Все, что нужно, будет сформулировано и получено в последующих разделах. Для доказательства ЗБЧ в 
п. 3 будет выведено биномиальное распределение вероятностей. Предварительно, в п.2, мы выведем формулу для биномиальных коэффичиентов, входящих в выражения для биномиальных вероятностей. Их наглядное представление дается с помощью треугольника Паскаля (французский математик, 1623-1662).

\section{2. Треугольник Паскаля и биномиальные коэффициенты}

Треугольник Паскаля (см. рисунок) это таблица, которая строится по следующему правилу. В вершине таблицы и в начале и конце каждой строки стоят единицы, а каждое число внутри таблицы

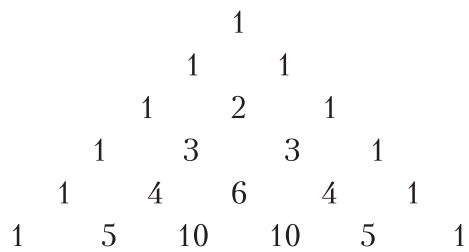

равно сумме двух чисел, стоящих над ним. Вам, конечно, знакомы выражения $a+b$, $(a+b)^{2}=a^{2}+2 a b+b^{2}$ и $(a+b)^{3}=a^{3}+$ $+3 a^{2} b+3 a b^{2}+b^{3}$. Тогда вы увидите, что числа в 1-й, 2-й и 3-й строках таблицы (вершина не в счет, считаем ее 0-й строкой) - это в точности коэффициенты, входящие в эти выражения. А если не поленитесь выписать разложения для $(a+b)^{4}$ и $(a+b)^{5}$, то увидите, что коэффициенты при произведениях $a$ и $b$ в разных степенях снова даются числами из треугольника Паскаля. Примем для них обозначение $C_{n}^{k}$ - это число, стоящее на $k$-м месте в $n$-й строке ${ }^{2}$ (снова считая, что единица в начале строки стоит на 0-й позиции). Например, $C_{3}^{0}=C_{3}^{3}=1, C_{3}^{1}=$ $=C_{3}^{2}=3$. Легко видеть также, что $C_{n}^{1}=$ $=C_{n}^{n-1}=n, n=1,2, \ldots$

Пользуясь этим обозначением, правило построения треугольника Паскаля можно

1 Те, кто знаком с началами комбинаторики, знают, что $C_{n}^{k}$ - это число сочетаний из $n$ по $k$, но здесь комбинаторные методы не используются. записать в виде

$$
\begin{aligned}
C_{n}^{k}=C_{n-1}^{k-1} & +C_{n-1}^{k}, \\
n & =2,3, \ldots, \quad k=1,2, \ldots, n .
\end{aligned}
$$

С помощью рекуррентного соотношения (1) докажем, что числа $C_{n}^{k}$, возникающие в треугольнике Паскаля, действительно являются биномиальными коэффициентами, т.е. коэффициентами в выражениях для степеней $(a+b)^{n}$ бинома $a+b$. Понятно, что такое выражение имеет вид

$$
\begin{aligned}
& (a+b)^{n}= \\
& =a^{n}+B_{n}^{1} a^{n-1} b+\ldots+B_{n}^{k} a^{n-k} b^{k}+\ldots+b^{n},
\end{aligned}
$$

где $B_{n}^{k}$ - некоторые коэффициенты, про которые мы хотим доказать, что $B_{n}^{k}=C_{n}^{k}$. (Удобно заметить, что верхний индекс при $B$ равен степени $b$ в том же слагаемом.)

Доказательство будет проводиться методом математической индукции. Выше мы убедились, что наше утверждение верно при $n=1$ и 2. Предположим теперь, что оно доказано для всех степеней до $n-1$ включительно, и докажем, что тогда оно верно и для степени $n$, т.е. что в разложении (2) $B_{n}^{k}=C_{n}^{k}$. Поскольку $(a+b)^{n}=$ $=(a+b)^{n-1}(a+b)$, посмотрим, как слагаемое $B_{n}^{k} a^{n-k} b^{k}$ в (2) происходит из слагаемых в аналогичном (2) разложении для степени $n-1$ при умножении на $a+b$. Оно получается при умножении на $a$ слагаемого, содержащего на единицу меньшую степень $a$, т.е. $a^{n-k-1} b^{k}$, и точно так же при умножении на $b$ слагаемого, содержащего $b^{k-1}$, т.е. $a^{n-k} b^{k-1}$. Указанные степени входят с коэффициентами $B_{n-1}^{k}$ и $B_{n-1}^{k-1}$ соответственно, но по предположению индукции $B_{n-1}^{k}=C_{n-1}^{k}$ и $B_{n-1}^{k-1}=C_{n-1}^{k-1}$. Поэтому из сказанного следует, что $B_{n}^{k}=$ $=C_{n-1}^{k}+C_{n-1}^{k-1}$, а тогда согласно (1) $B_{n}^{k}=C_{n}^{k}$, что и требовалось.

Теперь получим явную формулу для $C_{n}^{k}$. Точнее, выпишем ее и докажем, что она действительно дает выражение для $C_{n}^{k}$. А именно, утверждается, что

$$
C_{n}^{k}=\frac{n(n-1) \ldots(n-k+1)}{1 \cdot 2 \cdot \ldots \cdot k} .
$$


Используя широко применяемое в математике обозначение $n !=1 \cdot 2 \cdot \ldots \cdot n$ (читается «n факториал») и умножая числитель и знаменатель на $(n-k) !=1 \cdot 2 \cdot \ldots \cdot(n-k)$, получаем эквивалентное выражение

$$
C_{n}^{k}=\frac{n !}{k !(n-k) !} .
$$

Для доказательства этого равенства убедимся сначала, что числа, стоящие в начальных строках треугольника Паскаля, выражаются этой формулой. По формуле (4) находим

$$
\begin{aligned}
& C_{2}^{1}=\frac{2 !}{1 ! 1 !}=2, C_{3}^{1}=\frac{3 !}{1 ! 2 !}=3, \\
& C_{4}^{1}=\frac{4 !}{1 ! 3 !}=4, C_{4}^{2}=\frac{4 !}{2 ! 2 !}=6 .
\end{aligned}
$$

Кроме того, $C_{3}^{2}=C_{3}^{1}=3$ и $C_{4}^{3}=C_{4}^{1}=4$, потому что соответствующие выражения отличаются только перестановкой множителей в знаменателе. Полученные числа совпадают со значениями в треугольнике Паскаля. Далее для доказательства по индукции покажем, что $C_{n}^{k}$ выражается формулой (4), в предположении, что эта формула верна для $C_{n-1}^{k}$.

Действительно, пользуясь соотношением (1), имеем

$$
\begin{aligned}
C_{n}^{k} \stackrel{(1)}{=} & C_{n-1}^{k-1}+C_{n-1}^{k} \stackrel{(4)}{=} \\
& =\frac{(n-1) !}{(k-1) !(n-k) !}+\frac{(n-1) !}{k !(n-k-1) !}= \\
& =\frac{k(n-1) !+(n-k)(n-1) !}{k !(n-k) !}=\frac{n !}{k !(n-k) !},
\end{aligned}
$$

что и требовалось доказать.

Определение $n$ ! расширяют, формально полагая $0 !=1$. Тогда $C_{0}^{0}=C_{n}^{0}=1$, что позволяет распространить формулу (4) на крайние элементы треугольника Паскаля.

В следующем разделе вводятся вероятностные понятия, необходимые для доказательства ЗБЧ.

\section{3. Схема Бернулли и биномиальное распределение}

Схемой Бернулли (или испьтаниями Бернулли) называют случайный эксперимент, состоящий в проведении $n$ независи- мых повторных испытаний (игр, опытов, наблюдений) с двумя исходами, имеющими в этих испытаниях одни и те же вероятности.

Чтобы отвлечься от конкретики, считаем, что в каждом испытании происходит один из исходов 1 или 0 с вероятностями $p$ и $q=1-p$ соответственно (исход 1 будем называть «успехом»). В результате $n$ испытаний возникает цепочка $x_{1}, \ldots, x_{n}$, в которой $x_{k}=1$ или 0 в зависимости от исхода $k$-го испытания. Например, исходами четырех испытаний могут быть $(0,0,0,1)$ или $(1,1,0,1)$ и т.п. Каждую такую цепочку будем называть «элементарным исходом». Каждый элементарный исход (цепочка из нулей и единиц) может рассматриваться как запись некоторого $n$-значного числа в двоичной системе, откуда понятно, что число всевозможных элементарных исходов равно $2^{n}$.

Событием называется любое множество элементарных исходов, а вероятность события равна сумме вероятностей составляющих его элементарных исходов. Далее, случайной величиной называется любая численная величина, значение которой определяется элементарным исходом. Иначе говоря, случайная величина это функция на множестве элементарных исходов. В связи с ЗБЧ нас будет в первую очередь интересовать случайная величина $S_{n}$, равная числу успехов в $n$ испытаниях, и ее распределение вероятностей, т.е. набор вероятностей $P\left(S_{n}=k\right), k=0,1, \ldots, n$ (вероятности принято обозначать буквой $P$ от англ. probability). Указанная вероятность равна сумме вероятностей элементарных исходов, имеющих в своем составе $k$ единиц и $n-k$ нулей.

Подсчитаем вероятность такого события. Начнем с простого примера. Возможными исходами двух испытаний являются $(1,1),(1,0),(0,1)$ и $(0,0)$. Независимость испытаний означает, что вероятность совместного наступления каких-либо исходов двух испытаний равна произведению вероятностей наступления этих исходов в каждом из испытаний. Напомним, что исход 1 в каждом испытании происходит с 
вероятностью $p$ и исход 0 - с вероятностью $q=1-p$. Поэтому перечисленные исходы двух испытаний происходят с вероятностями $p^{2}, p q=q p$ и $q^{2}$. Точно так же вероятность произвольного элементарного исхода, содержащего $k$ единиц и $n-k$ нулей, равна $p^{k} q^{n-k}$.

Таким образом, элементарные исходы, входящие в событие $\left\{S_{n}=k\right\}$, имеют одну и ту же вероятность, равную $p^{k} q^{n-k}$, и остается подсчитать число этих элементарных исходов.

Покажем, что это число равно биномиальному коэффициенту $C_{n}^{k}$. Давайте выпишем разложение для $(a+b)^{n}$, не приводя подобные члены, не пользуясь перестановочностью произведения и не записывая произведения одинаковых сомножителей в виде степеней. Например,

$(a+b)^{2}=(a+b)(a+b)=a a+a b+b a+b b$.

Аналогично,

$$
(a+b)^{3}=(a+b)^{2}(a+b)=a a a+a b a+\ldots
$$

При увеличении степени на 1 число слагаемых будет удваиваться, для $n$-й степени их будет $2^{n}$ и они будут образовывать такие же цепочки из букв $a$ и $b$, как наши элементарные исходы - из единиц и нулей. Поэтому число элементарных исходов, содержащих $k$ единиц и $n-k$ нулей, будет равно числу слагаемых в разложении для $(a+b)^{n}$, содержащих $k$ множителей $a$ и $n-k$ множителей $b$, а как мы видели раньше, это число после приведения подобных членов и есть биномиальный коэффициент $C_{n}^{k}$.

С учетом сказанного получаем, что

$$
\begin{aligned}
P\left(S_{n}=k\right) & =C_{n}^{k} p^{k} q^{n-k}, \\
& n=1,2,3, \ldots, \quad k=0,1, \ldots, n
\end{aligned}
$$

Заметим, что

$$
\sum_{k=0}^{n} C_{n}^{k} p^{k} q^{n-k}=1
$$

поскольку, с одной стороны, это вероятность для $S_{n}$ принять любое из своих возможных значений, а с другой стороны, эта сумма представляет собой биномиальное разложение для $(p+q)^{n}=1$.
Итак, мы заготовили весь вероятностный аппарат, необходимый нам для доказательства ЗБЧ, которое будет проведено в следующем разделе.

\section{4. Закон больших чисел}

Как уже говорилось, этот закон гласит, что относительная частота успехов $S_{n} / n$ приближается к вероятности успеха $p$ при росте числа испытаний $n$. Точное вероятностное выражение этого утверждения дается формулой:

для любого $\varepsilon>0$

$$
P\left(\left|\frac{S_{n}}{n}-p\right|<\varepsilon\right) \rightarrow 1 \text { при } n \rightarrow \infty .
$$

Для всякого события $A$ вероятность $n p o^{-}$ тивоположного события $\bar{A}$, т.е. события, состоящего в невыполнении события $A$, равна $P(\bar{A})=1-P(A)$. В данном случае событие, противоположное событию под знаком вероятности в (6), состоит в выполнении противоположного неравенства. Поэтому ЗБЧ эквивалентен утверждению

$$
P\left(\left|\frac{S_{n}}{n}-p\right| \geq \varepsilon\right) \rightarrow 0 \text { при } n \rightarrow \infty .
$$

Наглядный смысл утверждения (6) состоит в том, что при больших $n$ относительная частота $S_{n}$ лежит в окрестности вероятности $p$ с большой (близкой к 1) вероятностью, а утверждения (7) - что $S_{n}$ выпадает из этой окрестности с малой вероятностью. Эти утверждения эквивалентны и доказывать можно любое из них.

Мы докажем утверждение (7) в простейшем случае, при $p=\frac{1}{2}$.

Теорема. $B$ схеме Бернулли с равновероятными исходами, когда $p=q=\frac{1}{2}$, для любого $\varepsilon>0$

$$
P\left(\left|\frac{S_{n}}{n}-\frac{1}{2}\right| \geq \varepsilon\right) \rightarrow 0 \text { nри } n \rightarrow \infty .
$$

Доказательство. По формуле (5) вероятность получения $k$ успехов в $n$ испытаниях в нашем случае, когда $p=\frac{1}{2}$, равна

$$
\begin{aligned}
P\left(S_{n}=k\right) & =C_{n}^{k} \cdot \frac{1}{2^{n}}, \\
n & =1,2,3, \ldots, \quad k=0,1, \ldots, n .
\end{aligned}
$$


Событие под знаком вероятности в (8) происходит тогда, когда $S_{n}$ равно какомунибудь из чисел $k$ таких, что

$$
\left|\frac{k}{n}-\frac{1}{2}\right| \geq \varepsilon .
$$

Поэтому вероятность в (7) равна сумме вероятностей (9) по всем таким $k$, и для доказательства теоремы надо показать, что эта сумма стремится к 0 при $n \rightarrow \infty$.

Множество значений $k$, удовлетворяющих (10), распадается на два множества: множество $K_{n, \varepsilon}$, состоящее из тех $k$, для которых

$$
\begin{aligned}
& \frac{k}{n}-\frac{1}{2} \leq-\varepsilon, \\
& \quad \text { т.е. } 0 \leq k \leq \frac{n}{2}-n \varepsilon=n\left(\frac{1}{2}-\varepsilon\right),
\end{aligned}
$$

и множество $K_{n, \varepsilon}^{\prime}$ тех $k$, для которых

$$
\begin{aligned}
& \frac{k}{n}-\frac{1}{2} \geq \varepsilon, \\
& \quad \text { т.е. } n \geq k \geq \frac{n}{2}+n \varepsilon=n\left(\frac{1}{2}+\varepsilon\right) .
\end{aligned}
$$

Ввиду симметрии биномиального распределения (9) попадание $S_{n}$ в каждое из этих множеств происходит с равными вероятностями, поэтому будем доказывать, что сумма вероятностей (9) по $k$, принадлежащим множеству $K_{n, \varepsilon}$, иначе говоря, удовлетворяющим неравенству (11), стремится к нулю.

Множество $K_{n, \varepsilon}$ имеет вид

$$
K_{n, \varepsilon}=\left\{0,1, \ldots, k_{n, \varepsilon}\right\}, k_{n, \varepsilon}=\left[n\left(\frac{1}{2}-\varepsilon\right)\right]
$$

([ $a]$ обозначает целую часть числа $a$, т.е. наибольшее целое число, не превосходящее $a$, например [2] $=2$, [1,4] = 1). В силу сказанного требуемое соотношение, из которого будет следовать теорема, имеет вид

$$
\begin{aligned}
& P\left(\frac{S_{n}}{n}-\frac{1}{2}<-\varepsilon\right)=\frac{1}{2^{n}} \sum_{k=0}^{k_{n, \varepsilon}} C_{n}^{k} \rightarrow 0 \\
& \text { при } n \rightarrow \infty \text { (14) } \\
& \text { (напомним, что согласно (9) биномиаль- } \\
& \text { ные вероятности при } \left.p=\frac{1}{2} \text { равны } C_{n}^{k} / 2^{n}\right) .
\end{aligned}
$$

Предположим, что $n$ нечетно (потом рассмотрим и четные $n$ ). В этом случае $n$-я строка треугольника Паскаля распадается на две симметричные части, например, 3-я строка - на 1, 3 и 3, 1, 5-я строка - на 1, 5, 10 и 10, 5, 1 и т.д. В общем случае такая «полустрока» содержит биномиальные коэффициенты $C_{n}^{k}$ с $k=0,1, \ldots, \frac{n-1}{2}$, всего $\frac{n+1}{2}$ членов.

Как уже отмечалось, сумма биномиальных коэффициентов (элементов треугольника Паскаля) по $n$-й строке равна $2^{n}$. Соответственно, сумма биномиальных вероятностей (9) по строке равна 1 , а сумма этих же вероятностей по полустроке равна $\frac{1}{2}$, т.е.

$$
P\left(\frac{S_{n}}{n}<\frac{1}{2}\right)=\frac{1}{2^{n}} \sum_{k=0}^{(n-1) / 2} C_{n}^{k}=\frac{1}{2} .
$$

Для доказательства (14) мы оценим отношение последнего слагаемого в сумме в (14), отвечающего $k=k_{n, \varepsilon}$, к последнему слагаемому в сумме в (15), отвечающему $k=\frac{n-1}{2}$, затем отношение предпоследнего слагаемого $\left(k=k_{n, \varepsilon}-1\right)$ в (14) к предпоследнему слагаемому в (15) $\left(k=\frac{n-1}{2}-1\right)$, затем отношение $3-\mathrm{x}, 4-\mathrm{x}$ слагаемых и т.д. Обнаружится, что эти отношения при всех $k=0,1, \ldots, k_{n, \varepsilon}$ ограничены одним и тем же числом, зависящим от $n$ и $\varepsilon$, которое стремится к нулю при каждом $\varepsilon>0$, когда $n \rightarrow \infty$.

Заметим, что число слагаемых в сумме (14) меньше, чем в сумме (15). Поэтому наш процесс сравнения слагаемых остановится на каком-то положительном значении $k$, которое мы не будем конкретизировать. Будем называть сумму от этого значения до $k=\frac{n-1}{2}$ (как в (15)) неполной суммой (15). В нее входит столько же слагаемых, как и в (14), и численное значение ее (с учетом множителя $\frac{1}{2^{n}}$ ) меньше $\frac{1}{2}$. Следовательно, отношение сум- 
мы (14) к неполной сумме ограничено тем же числом, стремящимся к нулю при $n \rightarrow \infty$, а поскольку неполная сумма вероятностей (15) меньше $\frac{1}{2}$, сумма вероятностей (14) стремится к нулю.

Итак, выпишем отношение последней в сумме (14) вероятности, отвечающей $k=k_{n, \varepsilon}$, к последней в (15) вероятности, отвечающей $k=\frac{n-1}{2}$. Поскольку по формуле (9) множитель $\frac{1}{2^{n}}$ у них общий, это отношение по формуле (4) равно

$$
\frac{C_{n}^{k_{n, \varepsilon}}}{C_{n}^{(n-1) / 2}}=\frac{n !}{k_{n, \varepsilon} !\left(n-k_{n, \varepsilon}\right) !} \cdot \frac{\left(\frac{n-1}{2}\right) !\left(\frac{n+1}{2}\right) !}{n !} .
$$

Само собой, $n$ ! сокращается, а остальное запишем как произведение двух дробей, которые будем оценивать. А именно,

$$
\frac{C_{n}^{k_{n, \varepsilon}}}{C_{n}^{(n-1) / 2}}=\frac{\left(\frac{n-1}{2}\right) !}{k_{n, \varepsilon} !} \cdot \frac{\left(\frac{n+1}{2}\right) !}{\left(n-k_{n, \varepsilon}\right) !}=\frac{A}{B}
$$

(т.е. $A$ обозначает первую дробь, а $B-$ обратную ко второй). Поскольку $k_{n, \varepsilon}=\left[n\left(\frac{1}{2}-\varepsilon\right)\right]<\frac{n-1}{2}$ (так как при достаточно больших $n$ имеем $\varepsilon n>\frac{1}{2}$ ), числитель в $A$ «длиннее», чем знаменатель, поэтому после сокращения множителей, входящих в $k_{n, \varepsilon} !$, получаем

$$
A=\left(k_{n, \varepsilon}+1\right)\left(k_{n, \varepsilon}+2\right) \cdot \ldots \cdot \frac{n-1}{2} .
$$

Точно так же

$$
B=\frac{(n+3)}{2} \cdot \frac{(n+5)}{2} \cdot \ldots \cdot\left(n-k_{n, \varepsilon}\right) .
$$

Теперь мы воспользуемся простым соотношением, которое сформулируем в виде леммы.

Лемма. Для любых $0<a<b, c>0$ имеем

$$
\frac{a}{b}<\frac{a+c}{b+c} .
$$

Доказательство предоставляем читателю (приведите к общему знаменателю и сравните числители). Мы только проиллюстрируем это на простом примере: $\frac{1}{2}=\frac{4}{8}<\frac{5}{9}$.

Согласно (18) и (19), отношение $\frac{A}{B}$ представляется в виде произведения дробей

$$
\frac{A}{B}=\frac{k_{n, \varepsilon}+1}{\frac{n+3}{2}} \cdot \frac{k_{n, \varepsilon}+2}{\frac{n+5}{2}} \cdot \ldots \cdot \frac{\frac{n-1}{2}}{n-k_{n, \varepsilon}} .
$$

Каждая последующая дробь отличается от предыдущей прибавлением единицы в числителе и знаменателе, поэтому по лемме они возрастают, так что наибольшая из них - последняя, следовательно, $\frac{A}{B}$ не превосходит этой последней дроби $\frac{(n-1) / 2}{n-k_{n, \varepsilon}}$, возведенной в степень, равную числу сомножителей в (20).

Оценим эту последнюю дробь. Очевидно, что для всякого $0<a<n$

$$
[a] \leq a, n-[a] \geq n-a, \frac{1}{n-[a]} \leq \frac{1}{n-a} .
$$

Поэтому

$$
\frac{\frac{n-1}{2}}{n-k_{n, \varepsilon}}=\frac{\frac{n-1}{2}}{n-\left[n\left(\frac{1}{2}-\varepsilon\right)\right]} \leq \frac{\frac{n}{2}}{\frac{n}{2}+n \varepsilon}=\frac{1}{1+2 \varepsilon} .
$$

Оценим теперь число сомножителей в (20). Оно равно числу множителей в (18), образующих $A$, а это есть число множителей, оставшихся от деления $\left(\frac{n-1}{2}\right)$ ! на $k_{n, \varepsilon}$ !, и оно равно $\frac{n-1}{2}-k_{n, \varepsilon}$. Пользуясь аналогом второго неравенства в (21), получаем

$$
\begin{aligned}
\frac{n-1}{2}-k_{n, \varepsilon}= & \frac{n-1}{2}-\left[\frac{n}{2}-n \varepsilon\right] \geq \\
& \geq \frac{n-1}{2}-\frac{n}{2}+n \varepsilon=n \varepsilon-\frac{1}{2} .
\end{aligned}
$$

Итак, из (22), (23) и сказанного выше об 
оценке $\frac{A}{B}$ следует, что

$$
\frac{A}{B} \leq\left(\frac{1}{1+2 \varepsilon}\right)^{n \varepsilon-\frac{1}{2}} \text {. }
$$

Напомним, что этим самым мы оценили отношение «крайней правой» биномиальной вероятности в сумме (14) к «крайней правой» вероятности в сумме (15). Теперь сделаем шаг влево и оценим отношение предпоследних вероятностей в этих суммах. Аналогично (16), (17) это отношение равно

$$
\frac{A_{1}}{B_{1}}=\frac{C_{n}^{k_{n, \varepsilon}-1}}{C_{n}^{(n-3) / 2}}=\frac{\left(\frac{n-3}{2}\right) !}{\left(k_{n, \varepsilon}-1\right) !} \cdot \frac{\left(\frac{n+3}{2}\right) !}{\left(n-k_{n, \varepsilon}+1\right) !} .
$$

Аналогично (18) имеем

$$
\frac{A_{1}}{B_{1}}=\frac{k_{n, \varepsilon}}{\frac{n+1}{2}} \cdot \frac{k_{n, \varepsilon}+1}{\frac{n+3}{2}} \cdot \ldots \cdot \frac{\frac{n-3}{2}}{n-k_{n, \varepsilon}-1} .
$$

Каждая дробь в (26) отличается от соответствующей дроби в (20) вычитанием 1 в числителе и знаменателе. По той же лемме получаем, что

$$
\frac{A_{1}}{B_{1}} \leq \frac{A}{B} \leq\left(\frac{1}{1+2 \varepsilon}\right)^{n \varepsilon-\frac{1}{2}} .
$$

То же самое произойдет и при всех последующих шагах влево: отношение соответствующих биномиальных вероятностей будет при каждом шаге уменьшаться и оставаться ограниченным той же величиной

$$
\left(\frac{1}{1+2 \varepsilon}\right)^{n \varepsilon-\frac{1}{2}}=(1+2 \varepsilon)^{-1 / 2}\left[\left(\frac{1}{1+2 \varepsilon}\right)^{\varepsilon}\right]^{n} .
$$

Поэтому интересующая нас сумма биномиальных вероятностей в (14) не превосходит неполной суммы вероятностей (15), меньшей $\frac{1}{2}$, умноженной на верхнюю границу (27). Последняя представлена в (27) в виде произведения постоянного числа (не зависящего от $n$ ) и члена геометрической прогрессии со знаменателем, меньшим 1, который, как известно, стремится к нулю при $n \rightarrow \infty$. Тем самым, теорема доказана в предположении, что $n$ нечетно.

Для доказательства теоремы в случае четного $n$ нужно центральное значение в треугольнике Паскаля просто выбросить и рассматривать биномиальные вероятности, отвечающие остальным значениям. Тогда сумма биномиальных вероятностей по неполной полустроке меньше $\frac{1}{2}$, что нисколько не мешает получению стремящейся к нулю верхней границы. Доказательство, повторяющее проведенное выше, предоставляем читателю.

\section{Литература}

1. Я.Бернулли. О законе больших чисел. М.: Наука, 1986.

2. Ю.Н.Тюрин, А.А.Макаров, И.Р.Высоикий, И.В.Ященко. Теория вероятностей и статистика. - М.: МЦНМО, 2014.

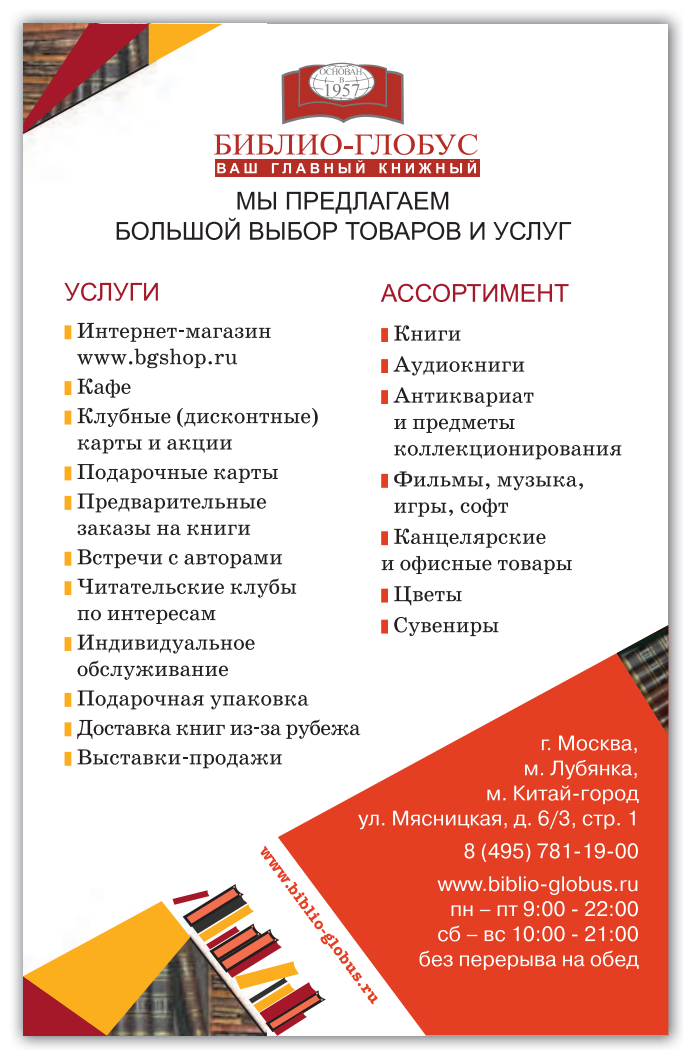

